Retrospective Analysis

\title{
Can the Novel Lumbo-Iliac Triangle Technique Based on Biplane Oblique Fluoroscopy Facilitate Transforaminal Percutaneous Endoscopic Lumbar Discectomy for Patients with L5-S1 Disc Herniation Combined with High Iliac Crest? A Case-Control Study of 100 Patients
}

\author{
Jun-Song Yang, MD', Kai-Xuan Liu, MD², Praveen Kadimcherla, MD², Lei Chu, MD³, \\ Zhong-Liang Deng, MD³, Yun-Kai Chan, MD, Peng Liu, MD'1, Tuan-Jiang Liu, MD', and \\ Ding-Jun Hao, MD ${ }^{1}$
}

\begin{abstract}
From: ${ }^{1}$ Department of Spine Surgery, Honghui Hospital, Xi'an Jiaotong University, Xi'an, Shaanxi, China; ${ }^{2}$ Atlantic Spine Center, West Orange, NJ, USA; 3Department of Orthopaedics, The Second Affiliated Hospital of Chongqing Medical University, Chongqing, China; ${ }^{4}$ Division of Neurosurgery, Department of Surgery, MacKay Memorial Hospital, Taipei City, Taiwan
\end{abstract}

Address Correspondence: Ding-Jun Hao, MD Department of Spine Surgery, Honghui Hospital, X'an Jiaotong University, No. 76 Nanguo Rd, Xi'an, Shaanxi, 710054, China E-mail: dingjun.hao@qq.com

Disclaimer: Jun-Song Yang and Kai-Xuan Liu contributed equally to this study. There was no external funding in the preparation of this manuscript.

Conflict of interest: Each author certifies that he or she, or a member of his or her immediate

family, has no commercial association (i.e., consultancies, stock ownership, equity interest, patent/licensing arrangements, etc.) that might pose a conflict of interest in connection with the submitted manuscript.

Manuscript received: 09-15-2019 Revised manuscript received: 11-06-2019 Accepted for publication: 11-14-2019

Free full manuscript: www.painphysicianjournal.com
Background: Percutaneous endoscopic lumbar discectomy (PELD) via the transforaminal approach is difficult at L5-S1 in patients presenting with high iliac crests (HIC). The conventional wisdom is that measurement using lumbar radiography, computed tomography (CT), or magnetic resonance imaging (MRI) is necessary.

Objectives: The objective of this study was to introduce a lumbo-iliac triangular (LI-Tri) technique based on biplane oblique fluoroscopy and verify whether it facilitated transforaminal PELD for patients with L5-S1 lumbar disc herniation (LDH) combined with HIC.

Study Design: A retrospective analysis.

Setting: All data were from Honghui Hospital in Xi'an.

Methods: One hundred patients with L5-S1 LDH combined with HIC were treated with PELD The LI-Tri technique was used in the first 50 patients (applied group). The other 50 patients were classified as the nonapplied group, in which the conventional technique was performed. Clinical outcome evaluation included Oswestry Disability Index (ODI) and Visual Analog Scale (VAS) scores. The intervals of follow-up were scheduled at 1 day and 1, 3, 6, 12, and 24 months postoperatively.

Results: No significant difference was observed with respect to demographic information $(P<$ .05 , respectively). There were 8 patients in the nonapplied group with difficult punctures. Together with the remaining 50 patients, the puncture was successful with the LI-Tri technique. The mean operative duration was shorter in the applied group (55 vs $70 \mathrm{~min}, P<.01$ ). Compared to the preoperative data, only the back pain VAS and ODI in the nonapplied group were nonsignificantly lower at one day postoperatively $(P>.05$, respectively). With the exception of the back pain VAS and ODI at one day postoperatively, no significant differences were observed in the 3 parameters at other time points postoperatively between 2 groups $(P>.05$, respectively).

Limitations: The study is limited by its retrospective, nonrandomized controlled design.

Conclusions: For patients with L5-S1 LDH combined with HIC treated by transforaminal PELD, the LI-Tri technique is simple and effective in preoperative evaluations, locating the skin entry point and guiding the puncture trajectory. Compared to the conventional technique, it shows advantages in terms of reducing intraoperative surgical duration and promoting fast postoperative recovery.

Key words: Endoscopic discectomy, iliac crest, L5-S1 disc, PELD, percutaneous, transforaminal.

Pain Physician 2020: 23:305-313 
$S$ ince Kambin (1) first introduced the concept of the triangular working zone into percutaneous posterolateral nucleotomy in 1973, percutaneous disc decompression performed with computed tomography (CT) and fluoroscopic guidance appears to be a safe and effective treatment for lumbar disc herniation (LDH) $(2,3)$. Although the surgery is less traumatic, it is not as intuitive as open surgery to confirm the decompression of the nerve root. The percutaneous endoscopic lumbar discectomy (PELD) technique has evolved with the development of surgical techniques and supporting equipment, which can achieve targeted decompression under a magnified and continuous clear visual field. As a bridge between conservative treatment and traditional open surgery, PELD is becoming the treatment of choice for LDH and even lumbar stenosis, achieving successful outcomes comparable to those of conventional open and microendoscopic surgery with less muscular trauma, faster function recovery, shorter hospital stays, and better preservation of spinal segment stability (411). According to the advantages of each technique in terms of addressing the herniated disc at different locations, the surgical approaches of PELD can be generally divided into transforaminal and interlaminar approaches. Transforaminal PELD is superior for removing central extruded disc and relieving the lateral exit zone stenosis due to intraforaminal herniation with or without foraminal stenosis. Several authors believe that PELD via the transforaminal approach is more difficult at L5-S1 and sometimes even at L4-L5 in patients presenting with hypertrophied facet joints and thickened transverse processes, especially high iliac crests (HIC) (12-17). Therefore, they performed the measurement using lumbar radiography, $\mathrm{CT}$, or magnetic resonance imaging (MRI) to evaluate the relation between the iliac crest and L5-S1 disc, and planned the puncture trajectory preoperatively (12-14). Nevertheless, these measurements were performed using a single plane (coronal or axial) $(12,13)$. In fact, the puncture trajectory should consider the biplane simultaneously, in which the end of the puncture needle should be properly tilted to the cephalic side while angulating with the horizontal plane. Furthermore, tedious measurements undoubtedly increase the difficulty of operation. Based on previous successful clinical experience in dealing with L5-S1 herniation accompanying HIC, we present a case-control study of 100 patients to introduce a novel lumbo-iliac triangular (LI-Tri) technique based on biplane oblique fluoroscopy and verify whether it facilitates transforaminal PELD for patients with L5-S1 disc herniation combined with HIC.

\section{Methods}

The definition of HIC is derived from Choi's (13) degree system, classifying the relationship between the location of the highest point of the iliac crest and the adjacent bony structures such as the L 5 pedicle, and the L4 inferior and S1 superior endplates. In our patient selection, the highest point of the iliac crest above the midpoint of the L5 pedicle (Type $>5$ ) was regarded as "HIC." Between January 2016 and December 2016, 100 patients with LDH combined with HIC were treated with PELD via a unilateral transforaminal approach in 2 tertiary spine centers. The LI-Tri technique was used in the first 50 patients (applied group) with L5-S1 disc herniation. The other 50 patients were classified as the nonapplied group, in which the conventional puncture process was performed $(11,17,18)$. The puncture location was considered unsatisfactory after 20 puncture attempts and regarded as a difficult puncture. If this occurred in the nonapplied group, the LI-Tri technique was introduced into the puncture process, as with the applied group. Three senior surgeons with experience in over 500 transforaminal PELD cases performed the surgeries. Approval to conduct the study was granted by the ethics committees of hospitals. The institutional review board approved the study, and informed consent and protocols were provided to all patients. Inclusion criteria were as follows: (a) clinical symptoms and signs of unilateral neurological deficit including radiculopathy, paresthesia, motor weakness that did not respond to conservative treatments including medication, physiotherapy, and nerve root blocks for 6 weeks; (b) symptoms corresponding to preoperative $\mathrm{MRI}$ and/or CT scans. Exclusion criteria were as follows: (a) definite segmental instability (anterior or posterior displacement $>3 \mathrm{~mm}$ or the angle change of the endplate $>15$ degrees on the dynamic radiography); (b) severe central canal stenosis (less than $10 \mathrm{~mm}$ ) on preoperative MRI or $\mathrm{CT}$; (c) cauda equina syndrome; (d) highly migrated nucleus pulposus beyond the low rims of adjacent pedicles; and (e) suspected infection or malignant diseases.

\section{Preoperative Preparation}

All patients were operated on under local anesthesia in the prone position. A Kambin frame was placed under the patient's abdomen to decrease the lumbar lordosis and maintain hip joint flexion. Intravenous dex- 
medetomidine $(0.5 \mu \mathrm{g} / \mathrm{kg}$ bolus, followed by $0.1-0.5 \mu \mathrm{g} /$ $\mathrm{kg} / \mathrm{h}$ ) was given to provide an ideal sedative effect and improve surgical tolerance.

\section{Surgical Process}

For the nonapplied group, the conventional puncture process was performed as described in previous studies $(11,17,18)$. The LI-Tri technique was based on a biplane oblique fluoroscopy, in which the $\mathrm{C}$-arm was rotated to the punctured side in the axial plane and tilted to the cranial side in the coronal plane (Figs. 1A and $\mathrm{C}$ ). Therefore, a lumbo-iliac triangle zone was visible on fluoroscopy, formed by the L5 inferior endplate (superior edge), the superior articular process (SAP) of $\mathrm{S} 1$ (medial edge), and the posterolateral margin of the iliac crest (lateral edge) (Fig. 1D); the center of the triangle is the targeted $\mathrm{L} 5-\mathrm{S} 1$ disc. When the projection point of the tip of the transverse process was located at the center between the projection point of the base of the spinous process and the anterior margin of the vertebral body, the surface projection point of the superior edge of the SAP at the oblique fluoroscopy was the skin entry point (Figs. 2A and B). Along with the projection angle of fluoroscopy or bull's eye projection, the needle was easily placed at the superior edge of the SAP or into the disc space. When the puncture needle was placed at the satisfactory position (Figs. $3 \mathrm{~A}$ and $\mathrm{B}$ ), sequential dilation was performed along with the guide wire. In some patients with accompanying foraminal stenosis, slight foraminoplasty was also performed. The working cannula was placed at the ventral side of the SAP. The surrounding adhesive soft tissue and ligament were released by radiofrequency (Ellman Trigger-Flex Probe ${ }^{\mathrm{TM}}$, Ellman International, Hewitt, NY). Together with the endoscope (SPINENDOS GmbH, Munich, Germany), it was rotated into the foramen cannula. When the lateral recess was widened, the attached capsule and hypertrophic ligament flavum were removed, and the epidural space was exposed. The ruptured fragment was detected when we levered the working cannula. When the protruded nucleus pulposus was thoroughly removed, the crevasse of the annulus fibrosus was located. Annuloplasty was performed to prevent recurrent herniation. Free-floating dura and nerve root in the irrigation fluid was a sign of satisfactory decompression. The skin incision was closed without drainage.

\section{Postoperative Management and Outcome Assessment}

To diminish the risk of recurrent disc herniation, a soft lumbar brace was recommended for approximately 4 weeks to ensure that the ruptured annular fibrosis could heal and relieve the postoperative muscle spasm. Clinical outcome evaluation included the Oswestry Disability Index (ODI) score and Visual Analog Scale (VAS) score without the usage of analgesic to truly

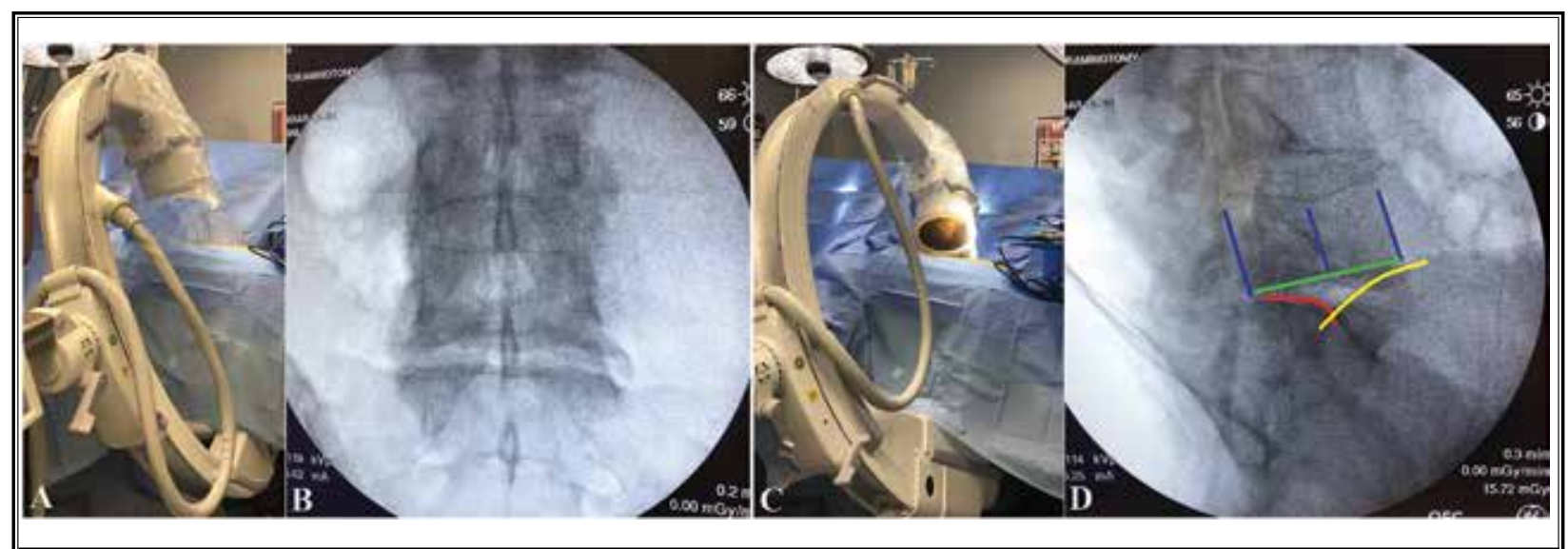

Fig. 1. The C-arm tilted to the cranial side (Panel A) in the coronal plane, making the adjacent endplates of L5-S1 disc overlap completely (Panel B); then further rotated to the punctured side in the axial plane (Panel C). When the projection point of the tip of the transverse process (middle blue line) was located at the center between the projection point of the base of the spinous process (posterior blue line) and the anterior margin of the vertebral body (anterior blue line), a triangle zone was visible on fluoroscopy (Panel D), formed by the L5 inferior endplate (superior edge, green line), the superior articular process (SAP) of $S 1$ (medial edge, red line), and the posterolateral margin of the iliac crest (lateral edge, yellow line). 


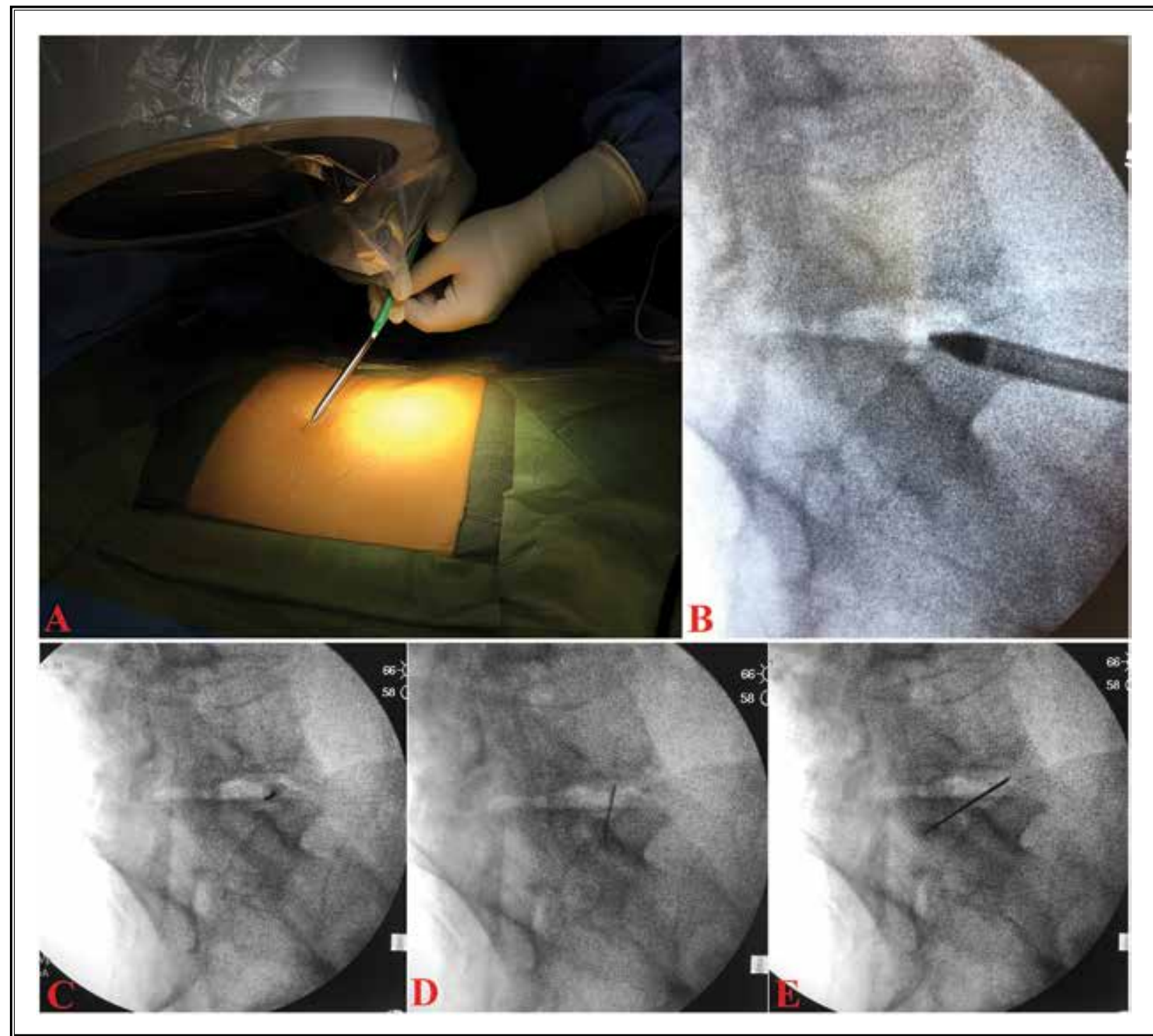

Fig. 2. The surface projection point (Panel B) of the superior edge of the SAP at the oblique fluoroscopy was the skin entry point (Panel A). As long as the fluoroscopic image of the puncture needle became a dot (Panel C), the puncture trajectory was ideal, which was consistent with the projection trajectory of the C-arm. Panel D shows that the tilted degree of the puncture needle was too large at the coronal plane. Panel E shows that the rotated degree of the puncture needle was too small at the axial plane.

reflect the intensity of back and leg pain. The physical examinations and clinical asessments were performed by another surgeon who did not participate in the surgical procedures. The intervals of follow-up were scheduled at 1 day and 1, 3, 6, 12, and 24 months after surgery. Relevant complications, including intraoperative conversion from conventional puncture to the LI-Tri technique, postoperative dysthesias, and motor weakness were also recorded. Postoperative MRI or CT ex- aminations were recommended, especially for patients without satisfactory symptom relief.

\section{Statistical Analysis}

Statistical analyses were performed using SPSS Version 17.0 (SPSS Inc., Chicago, IL). Measurement data were expressed as mean \pm standard deviation (SD). Preoperative and postoperative VAS scores for back and leg pain, as well as ODI scores, were analyzed 


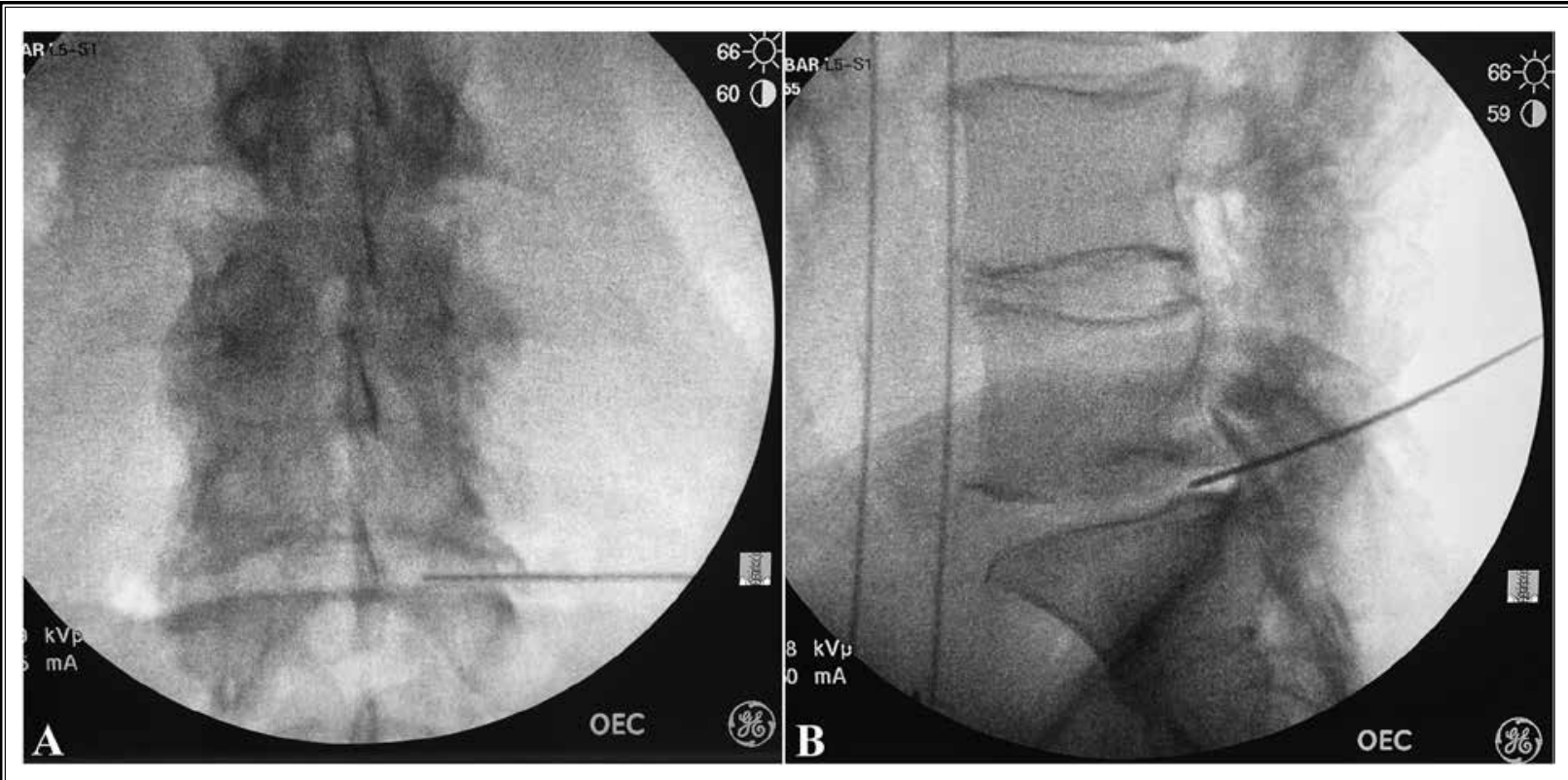

Fig. 3. The puncture needle was placed at the satisfactory position, which was confirmed by the anteroposterior (Panel A) and lateral fluoroscopy (Panel B).

using ANOVA retrospectively. $P<.05$ was considered significant.

\section{RESULTS}

The mean age of the patients was $37.5 \pm 10.5$ years in the applied group and $36.9 \pm 11.1$ years in the nonapplied group. The percentage of men was $82 \%$ in the applied group and $84 \%$ in the nonapplied group. No significant difference was observed with respect to demographic information $(P<.05$, respectively). While no cases required conversion to open procedures, there were 8 patients in the nonapplied group with difficult punctures, in which the LI-Tri technique was introduced. Together with the remaining 50 patients, the lumboiliac triangular zone was visible, meaning that the puncture was feasible despite the presence of HIC. The puncture was also successful in the 58 patients using the LI-Tri technique. The mean operative duration was shorter in the applied group (55 vs $70 \mathrm{~min}, P<.01$ ). Two patients experienced transient dysesthesia located at the exiting nerve in the nonapplied group, while no patients complained of dysesthesia in the applied group. No dural tears, motor weakness, or infections were observed in the entire cohort. Four patients underwent revision surgery after recurrence during follow-up: 2 in each group. Preoperative and postoperative VAS scores for low back pain and sciatica as well as ODI scores are summarized in Figs. 4-6. Compared to the preoperative data, only the back pain VAS and ODI in the nonapplied group were nonsignificantly lower at one day postoperatively ( $P>.05$, respectively). A significant improvement in VAS scores for back pain and sciatica and ODI scores were observed in other follow-up intervals $(P<$ .05 , respectively). With the exception of the back pain VAS and ODI at one day postoperatively, no significant differences were observed in the 3 parameters at other time points after surgery between these 2 groups $(P>$ .05 , respectively).

\section{Discussion}

In the nonapplied group, the so-called Tessys technique described by Schubert and Hoogland $(19,20)$ was applied, in which the puncture trajectory was toward the tip of the SAP. However, the puncture needle was targeted to the disc space in the applied group. Because the mean operative duration was shorter in the applied group, this suggests that the simplified LI-Tri technique is beneficial for control of surgical duration. The other advantage of guiding the puncture accurately made LI-Tri technique applicable to some cases that failed conventional puncture. When the lumbo-iliac triangle zone was visible, the puncture needle was successfully 

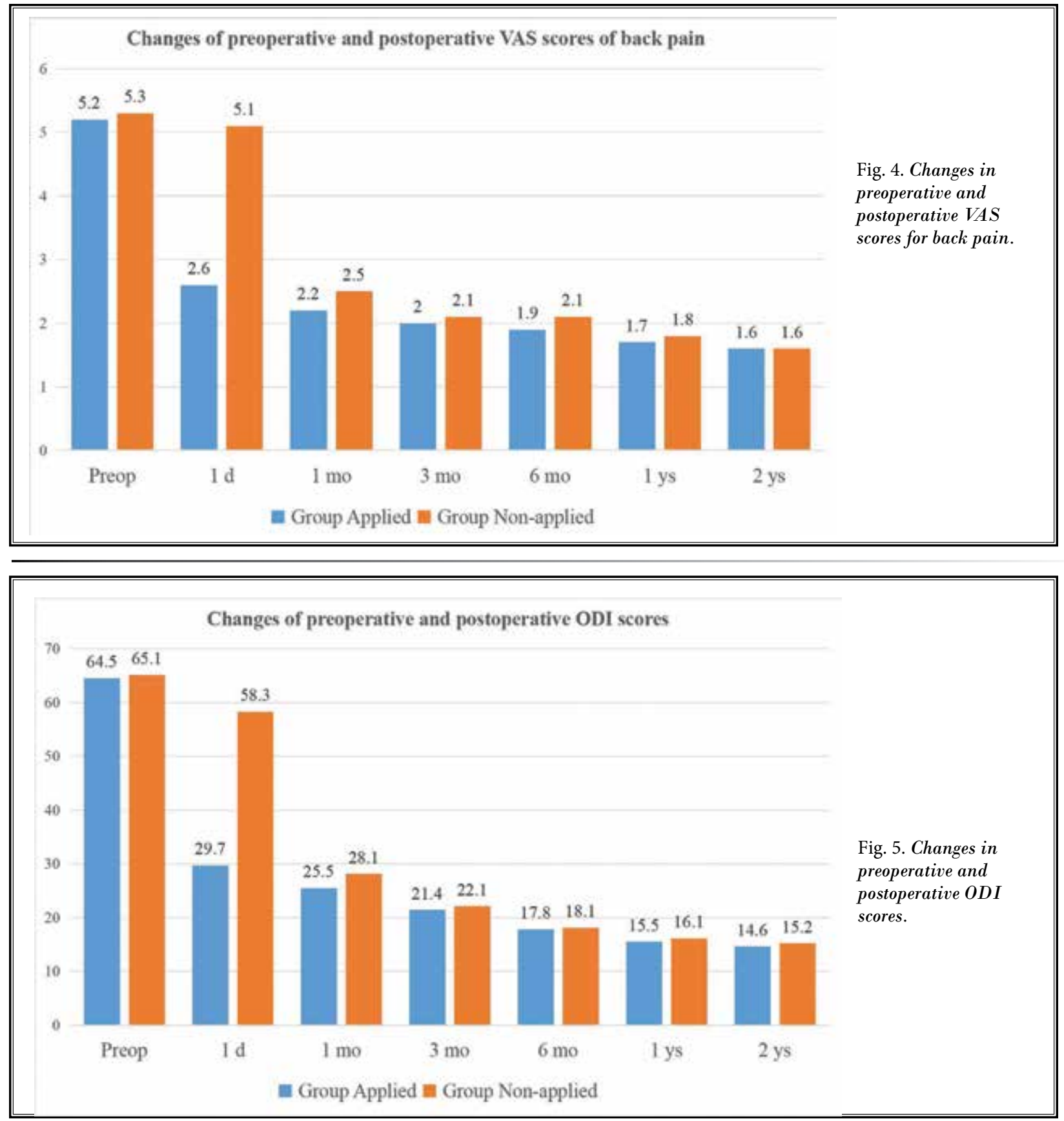

inserted in the 8 patients from the nonapplied group who had difficult puncture. Precise puncture needle insertion was beneficial for decreasing surgery-related complications. We believe that the 2 patients who experienced transient dysesthesia in the nonapplied group did so because of unsatisfactory placement of the puncture needle and the further working cannula.
When levering the working cannula, it can pinch the exiting nerve root and cause neural dysfunction. The repeated and prolonged puncture manipulation definitely induced severe damage to the back muscle.

Secondary hemorrhage and tissue edema are the reasons for low-back pain and functional limitations. This explains why the back VAS and ODI scores were 
Fig. 6. Changes in preoperative and postoperative VAS scores for sciatica.

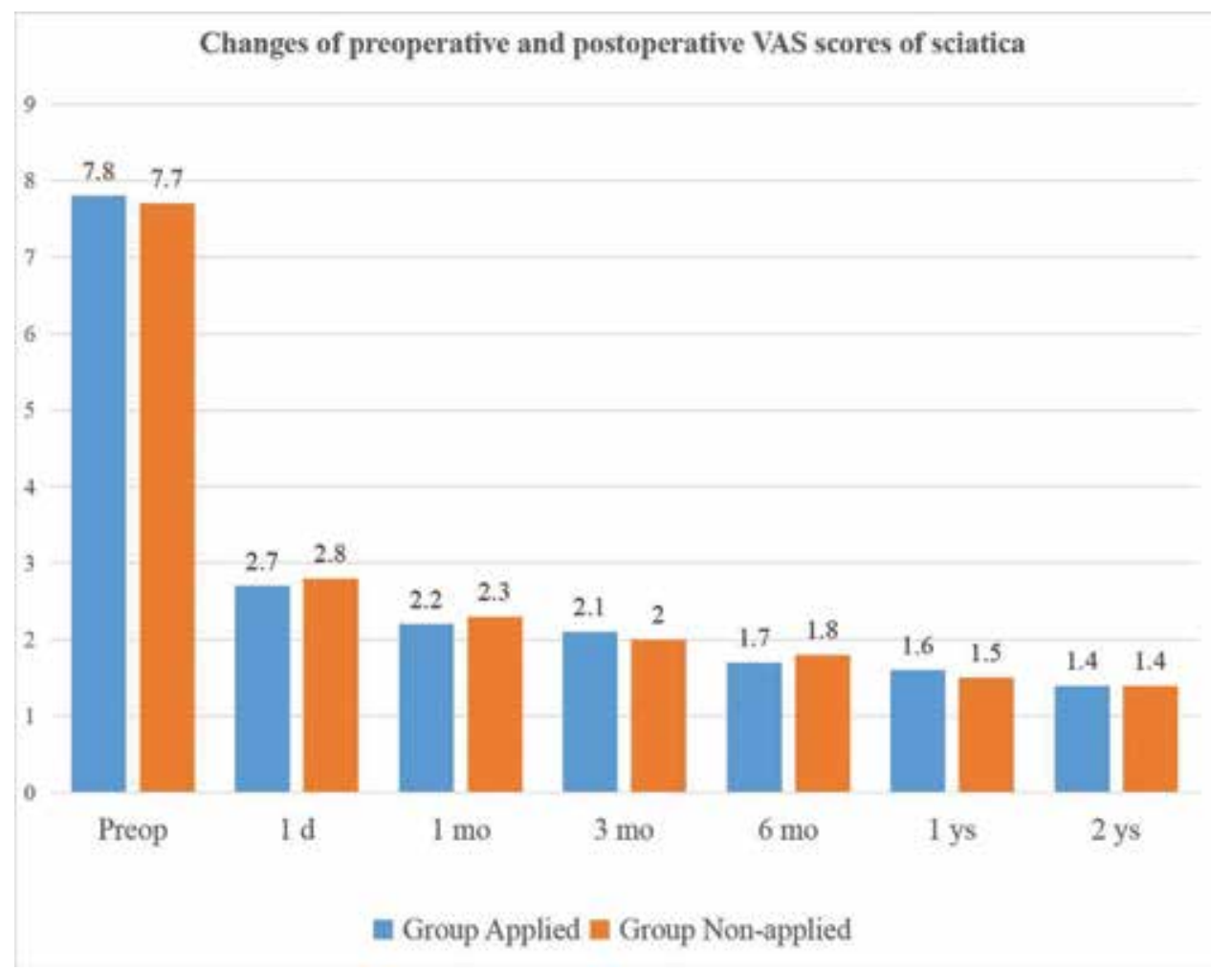

higher in the nonapplied group at one day after surgery. With the resorption of hematoma and regression of muscular edema, the difference between the 2 groups became insignificant. The degree of neural decompression directly determined the postoperative improvement of neural function. Thorough neural decompression was confirmed under endoscopy for both groups, explaining why there was no significant difference in the VAS scores for sciatica between the groups. Because the severity of pain was measured without painkiller, the score more objectively reflected clinical improvement.

HIC is considered a relative contraindication for transforaminal PELD at L5-S1. Several authors have made numerous explorations of anatomical classifications, radiological measurements, surgical approach modifications, and development of navigation equipment (12-18). Choi et al (13) performed a study to measure the 2-dimensional relationship between the iliac crest and the L5-S1 disc on lumbar radiography. While they provide a definition and classification of $\mathrm{HIC}$, the highest point of the iliac crest used in the classification is not actually the position of the puncture needle or working cannula contacting the ilium; thus it only indirectly reflects the relationship between the ilium and the puncture needle. This explains why the puncture is available in patients with HIC. Tezuka et al (12) studied 323 patients and assessed the 3-dimensional relationship between the transforaminal surgical trajectory and the iliac crest using abdominal CT scans. They found that the transforaminal puncture interfered with the iliac crest at L5-S1 in 99.7\% (right) and $100 \%$ (left) of patients. Therefore, a foraminoplasty was recommended. Nevertheless, this finding is not valuable for guiding the puncture process. Eun et al (14) introduced a preoperative trajectory evaluation method for L5-S1 transforaminal PELD using MRI or CT examinations. Skin markers are attached to the patient's back as a reference point at the preoperative MRI or CT. Depending on the trajectory of preoperative planning, the distance between the marker and the ideal puncture point is measured; then the skin entry point is located. However, localization based on 3-plane measurement requires a 3-dimensional spatial conversion and multiple distance measurements that may induce deviation in actual manipulation. Because the 
supine position is used during radiological examination, the intraoperative position alternation may also lead to deviation. More importantly, the technique can only determine the entry site, and cannot guide the puncture trajectory intraoperatively. To overcome the obstruction from the iliac crest, Choi (15) and Osman (16) reported an endoscopic transiliac approach. However, it is technically demanding to establish a hole of appropriate size and ideal position at the ilium. The complication of ilium fracture and bleeding are worrisome. To guide the puncture process, Fan et al (17) used an isocentric navigation technique in 11 difficult puncture cases at the L5/S1 level. Ao et al (18) presented a surgical technique for PELD assisted by O-arm-based navigation. Because an additional arch-guided device or costlier O-arm-based navigation was required, the popularity of this technique is limited.

Integrating the advantages of these techniques, our LI-Tri technique can be used for preoperative evaluation, location of the skin entry point, and guidance of the puncture trajectory. Actually, this technique has been used by pain doctors to access the L5-S1 disc for intradiscal interventions. Furthermore, it is really meaningful to introduce it into L5-S1 transforaminal discectomy rather than relying on complicated markings and costly imaging studies. The presence of the "triangle window" can be used to predict whether it is feasible to place the puncture needle into the targeted intervertebral foramen or disc. As the lateral edge of the lumbosacral triangle window, the posterolateral margin of the iliac crest is where the actual puncture needle and working cannula make contact with the ilium. In addition to the ilium, the hypertrophic SAP and/ or transverse process of $L 5$, even with collapse of the intervertebral space, and the bony structures impeding the puncture process, are intuitively visualized at the biplane oblique fluoroscopy that fuses the coronal and sagittal images simultaneously. Without additional CT or MRI examination or tedious measurements, the location of the skin entry point is based on real-time intraoperative fluoroscopy. When the C-arm is tilted to the cranial side, making the adjacent endplates of L5S1 disc overlap completely, it means that the angle of projection is completely parallel to the intervertebral space (Fig. 1B), and that the ideal trajectory at the sagittal plane addresses the interforaminal compression. Additionally, with the rotation of the C-arm, the intervertebral foramen parallel to the sagittal plane is visible, giving an intuitive visual view of the intervertebral foramen and the deep intervertebral space. When the projection point of the tip of transverse process is located at the center between the projection point of the base of the spinous process and the anterior margin of vertebral body, the rotation angle is approximately equal to the angle between the ideal puncture trajectory and the horizontal plane. Therefore, the projection trajectory of the C-arm is actually consistent with the puncture trajectory. As long as the fluoroscopic image of the puncture needle becomes a dot (Fig. 2CE), the puncture trajectory is ideal. Without additional navigation equipment required, this is beneficial for technique generalization and reduction in medical cost.

\section{Limitation}

The main limitation of our study is that it is a retrospective, nonrandomized controlled study. The C-arm rotation degree at the axial plane was based on the practitioner's experience, which can change depending on the location of herniation; the LI-Tri technique is only sufficient to guide the needle to reach the ventral surface of the SAP. Further foraminoplasty and the adjustment of the working cannula are helpful to establish the working channel at the ideal position.

\section{Conclusion}

For patients with L5-S1 disc herniation combined with HIC treated by transforaminal PELD, the LI-Tri technique is simple and effective in preoperative evaluations, locating the skin entry point and guiding the puncture trajectory. Compared to the conventional puncture technique, it shows advantages in terms of reducing intraoperative surgical duration and promoting fast postoperative recovery.

\section{Author Contributions}

Guarantors of integrity of entire study, Hao DJ, Liu KX, Deng ZL; study concepts/study design or data acquisition or data analysis/interpretation, all authors; manuscript drafting or manuscript revision for important intellectual content, all authors; manuscript final version approval, all authors; literature research, Liu P; clinical studies, Liu KX, Kadimcherla P, Yang JS; statistical analysis, Liu TJ, Chan YK, Chu L; and manuscript editing, all authors. 


\section{REFERENCES}

1. Kambin P, Sampson S. Posterolateral percutaneous suction-excision of herniated lumbar intervertebral discs. Clin Orthop 1986; 207:37-43.

2. Gangi A, Dietemann JL, Ide C, Brunner P, Klinkert A, Warter JM. Percutaneous laser disk decompression under CT and fluoroscopic guidance: Indications, technique, and clinical experience. Radiographics 1996; 16:89-96.

3. Erginousakis D, Filippiadis DK, Malagari A, et al. Comparative prospective randomized study comparing conservative treatment and percutaneous disk decompression for treatment of intervertebral disk herniation. Radiology 2011; 260:487-493.

4. Hermantin FU, Peters T, Quartarato LA. A prospective, randomized study comparing the results of open discectomy with those of video-assisted arthroscopic microdiscectomy. J Bone Joint Surg 1999; 81:958-965.

5. Choi KC, Lee JH, Kim JS, et al. Unsuccessful percutaneous endoscopic lumbar discectomy: A single-center experience of 10,228 cases. Neurosurgery 2015; 76:372-380; discussion 380-381; quiz 381.

6. Ruetten S, Komp M, Merk H, Godolias G. Surgical treatment for lumbar lateral recess stenosis with the fullendoscopic interlaminar approach versus conventional microsurgical technique: A prospective, randomized, controlled study. J Neurosurg Spine 2009; 10:476-485.

7. Lee DY, Shim CS, Ahn Y, Choi YG, Kim HJ, Lee $\mathrm{SH}$. Comparison of percutaneous endoscopic lumbar discectomy and open lumbar microdiscectomy for recurrent disc herniation. J Korean Neurosurg Soc 2009; 46:515-521.

8. Ahn Y, Lee SH, Lee JH, Kim JU, Liu WC. Transforaminal percutaneous endoscopic lumbar discectomy for upper lumbar disc herniation: Clinical outcome, prognostic factors, and technical consideration. Acta Neurochir 2009; 151:199-206.

9. Birkenmaier $C$, Komp $M$, Leu HF, Wegener B, Ruetten S. The current state of endoscopic disc surgery: Review of controlled studies comparing full-endoscopic procedures for disc herniations to standard procedures. Pain Physician 2013; 16:335-344.

10. Lee CW, Yoon KJ, Ha SS, Kang JK. Foraminoplastic superior vertebral notch approach with reamers in percutaneous endoscopic lumbar discectomy: Technical note and clinical outcome in limited indications of percutaneous endoscopic lumbar discectomy. J Korean Neurosurg Soc 2016; 59:172-181.

11. Ahn Y, Oh HK, Kim H, Lee SH, Lee HN. Percutaneous endoscopic lumbar foraminotomy: An advanced surgical technique and clinical outcomes. Neurosurgery 2014; 75:124-133; discussion 132-133.

12. Tezuka F, Sakai T, Abe M, et al. Anatomical considerations of the iliac crest on percutaneous endoscopic discectomy using a transforaminal approach. Spine ] 2017; 17:1875-1880.

13. Choi KC, Park CK. Percutaneous endoscopic lumbar discectomy for $\mathrm{L}_{5-}$ $\mathrm{S} 1$ disc herniation: Consideration of the relation between the iliac crest and $\mathrm{L}_{5}-\mathrm{S}_{1}$ disc. Pain Physician 2016; 19:E301-E308.

14. Eun SS, Lee SH, Liu WC, Erken HY. A novel preoperative trajectory evaluation method for $\mathrm{L}_{5}-\mathrm{S}_{1}$ transforaminal percutaneous endoscopic lumbar discectomy. Spine ] 2018; 18:1286-1291.

15. Choi G, Kim JS, Lokhande P, Lee SH. Percutaneous endoscopic lumbar discectomy by transiliac approach: A case report. Spine (Phila Pa 1976) 2009; 34:E443-E446.

16. Osman SG, Marsolais EB. Endoscopic transiliac approach to $\mathrm{L}_{5}-\mathrm{S}_{1}$ disc and foramen. A cadaver study. Spine (Phila Pa 1976) 1997; 22:1259-1263.

17. Fan G, Wang T, Hu S, Guan X, Gu X, He $S$. Isocentric navigation of percutaneous endoscopic transforaminal discectomy at the $L_{5} / S_{1}$ level in difficult puncture cases: A technical note. Pain Physician 2017; 20:E531-E540.

18. Ao S, Wu J, Tang Y, et al. Percutaneous endoscopic lumbar discectomy assisted by $\mathrm{O}$-arm-based navigation improves the learning curve. Biomed Res Int 2019; 2019:6509409.

19. Schubert M, Hoogland T. Endoscopic transforaminal nucleotomy with foraminoplasty for lumbar disk herniation. Oper Orthop Traumatol 2005; 17:641-661.

20. Hoogland $T$, Schubert M, Miklitz B, Ramirez A. Transforaminal posterolateral endoscopic discectomy with or without the combination of a low-dose chymopapain: A prospective randomized study in 280 consecutive cases. Spine 2006; 31:E890-E897. 
